\title{
Prescribed Renoprotective Chinese Herbal Medicines Were Associated with a Lower Risk of All-Cause and Disease-Specific Mortality among Patients with Chronic Kidney Disease: A Population-Based Follow-Up Study in Taiwan
}

\author{
Chuan-Fa Hsieh, ${ }^{1,2}$ Huan-Cheng Chang, ${ }^{3,4}$ Song-Lih Huang, ${ }^{5}$ Chien-Lung Chen, ${ }^{3,6}$ \\ Wei-Ta Chen, ${ }^{7}$ and Chen-Chang Yang ${ }^{8,9}$ \\ ${ }^{1}$ Department of Medical Education and Research, Taiwan Landseed Hospital, No. 77, Guangtai Rd., \\ Pingzhen Dist., Taoyuan 32449, Taiwan \\ ${ }^{2}$ Center for General Education, Hsin Sheng College of Medical Care and Management, No. 418, \\ Zhongxing Rd., Longtan Dist., Taoyuan 32555, Taiwan \\ ${ }^{3}$ Division of Nephrology, Department of Medicine, Taiwan Landseed Hospital, No. 77, Guangtai Rd., \\ Pingzhen Dist., Taoyuan 32449, Taiwan \\ ${ }^{4}$ Department of Health Care Management, Chang Gung University, No. 259, Wenhua 1st Rd., \\ Guishan Dist., Taoyuan 33302, Taiwan \\ ${ }^{5}$ Institute of Public Health \& Department of Public Health, School of Medicine, National Yang-Ming University, \\ No. 155, Sec. 2, Linong St., Taipei 11221, Taiwan \\ ${ }^{6}$ Institute of Systems Biology and Bioinformatics, Department of Biomedical Sciences and Engineering, National Central University, \\ No. 300, Zhongda Rd., Zhongli District, Taoyuan 32001, Taiwan \\ ${ }^{7}$ Department of Chinese Medicine, Taiwan Landseed Hospital, No. 77, Guangtai Rd., Pingzhen Dist., Taoyuan 32449, Taiwan \\ ${ }^{8}$ Institute of Environmental \& Occupational Health Sciences, School of Medicine, National Yang-Ming University, \\ No. 155, Sec. 2, Linong St., Taipei 11221, Taiwan \\ ${ }^{9}$ Division of Clinical Toxicology \& Occupational Medicine, Department of Medicine, Taipei Veterans General Hospital, \\ No. 201, Sec. 2, Shipai Rd., Taipei 11217, Taiwan
}

Correspondence should be addressed to Chen-Chang Yang; ccyang@vghtpe.gov.tw

Received 10 February 2017; Accepted 6 June 2017; Published 17 July 2017

Academic Editor: Evan P. Cherniack

Copyright (C) 2017 Chuan-Fa Hsieh et al. This is an open access article distributed under the Creative Commons Attribution License, which permits unrestricted use, distribution, and reproduction in any medium, provided the original work is properly cited.

Chinese herbal medicines (CHMs) containing aristolochic acid (AA) are associated with chronic kidney disease (CKD), but some prescribed CHMs have been shown to possess renoprotective effects. We conducted a nationwide retrospective cohort study to delineate the role of prescribed CHMs on the CKD progression. Renoprotective CHM (RPCHM) was defined if a CHM contained dong chong xia cao (Cordyceps sinensis (Berk.) Sacc.), da huang (Rheum palmatum L), huang qi (Astragalus membranaceus), dan shen (Salvia miltiorrhiza Bge.), and dong quai (Angelica sinensis (Oliv.) Diels) or belonged to specific mixture herbal formulations (Yishen capsule, Saireito, or Wen Pi Tang). Subjects who had ever used AA-containing CHMs, had cancer or HIV prior to CKD diagnosis, or died within the first month of CKD diagnosis were excluded. A total of 11,625 patients were eligible subjects. The adjusted hazard ratio (aHR) for all-cause mortality was $0.6(p<0.001)$ and $0.6(p=0.013)$ among subjects receiving RPCHMs containing Angelica sinensis and those receiving other RPCHMs, respectively. For CKD-related mortality, the aHR among subjects receiving RPCHMs containing Angelica sinensis was $0.6(p=0.025)$. The use of specific RPCHMs, especially those that contained Angelica sinensis, was associated with a lower risk of mortality among CKD patients. 


\section{Introduction}

Chinese herbal medicine (CHM) is one of the alternative therapies that are commonly used worldwide. It is estimated that approximately $20 \%$ of adults in the United States are taking herbal medicine [1], and the global use of herbal medicine products has increased significantly [2]. However, many studies have found that certain $\mathrm{CHMs}$, primarily those that contain aristolochic acid (AA), are associated with acute renal failure, urinary tract cancer $[3,4]$, and increased risk of chronic kidney disease (CKD) [5]. This has led to the misnomer of "Chinese herb nephropathy" in some English literature [6]. However, not all CHMs are nephrotoxic.

In fact, some CHMs such as dong quai (Angelica sinensis (Oliv.) Diels), huang qi (Astragalus membranaceus), and dong chong xia cao (Cordyceps sinensis (Berk.) Sacc.) have been shown to possess beneficial effects in the treatment of patients with CKD or to slow the progression of CKD in animal experiments $[7,8]$.

Taiwan is one of the countries with the highest incidence and prevalence of end-stage renal disease (ESRD) in the world [9]. The use of CHMs is fairly common in this country, even among those patients with CKD and subsequent ESRD $[10,11]$. While most prior studies in Taiwan revealed a positive association between the use of CHMs and the risk of CKD $[3,5,12]$, recent studies have demonstrated the potential protective effects of CHMs on the progression of CKD $[11,13$, 14]. The aim of this study therefore is to delineate the possible beneficial role of various non-AA prescribed CHMs on the progression of CKD among patients with incident CKD by employing a retrospective cohort study of CKD patients in Taiwan.

\section{Materials and Methods}

2.1. Study Population. The mandatory National Health Insurance (NHI) program in Taiwan was implemented in 1995 and covered more than $96 \%$ of the 22.5 million Taiwanese as of 2002. All treatment information of NHI enrollees, including medical diagnoses according to the International Classification of Diseases, Ninth Revision (ICD-9-CM), hospital admissions, emergency department and outpatient visits, and the prescription of both conventional medicines and CHMs, is systematically collected and entered into the National Health Insurance Research Database (NHIRD) that is accessible for research purposes.

We employed the Longitudinal Health Insurance Database (LHID2000), which was created by the NHI Bureau in 2000 and comprises one million randomly sampled enrollees who were insured under the NHI program in 2000. LHID2000 contains follow-up medical information for all NHI enrollees included in the database spanning from 1997 to 2008, and many papers have been published using this database $[14,15]$. All records were anonymized and deidentified prior to statistical analysis.

2.2. Eligible Participants and Duration of Follow-Up. Using the LHID2000, we searched for the following ICD-9CM diagnostic codes for all outpatient visits or hospital admissions to identify potential cases of CKD: 581-582, 585-589, 250.4, 274.1, 403, and 404. Because A codes were also used in Taiwan before 2002, we included the following codes in the identification of CKD: A350, A359.09, A359.10, and A358.27. CKD patients were defined as having had more than three consecutive outpatient visits (which is the same as approximately 3 months of follow-up) or hospitalized due to CKD. We selected these codes after considering the definition of $\mathrm{CKD}$ in several previously published papers that also employed the NHIRD [11, 12, 16, 17].

To ensure that only first-time diagnosis of CKD was included, we excluded subjects with a diagnosis of $\mathrm{CKD}$ between 1997 and 1999. We also excluded patients with preexisting cancer or human immunodeficiency virus (HIV) infection. Subjects without Taiwanese citizenship were also excluded, as well as those who had ever received CHMs containing AA because of its well-known nephrotoxicity and carcinogenicity $[4,12]$. Finally, to minimize the possibility of confounding by contraindication, we excluded patients who died within one month after the diagnosis of CKD because they were less likely to receive CHMs due to their severe underlying diseases or even moribund state [11].

The following AA-containing CHMs were identified using the standards for prescription recommended by the Committee on Chinese Medicine and Pharmacy in Taiwan: Ma-Dou-Ling (Fructus aristolochiae), Tian-Xian-Teng (Caulis aristolochiae), Xi-Xin (Asarum heterotropoides), GuanMu-Tong (Aristolochia manshuriensis), Guang-Fangchi (A. fangchi), Qing-Mu-Xiang (Radix aristolochiae), Mu-Tong (Akebia sp.), Fangchi (Stephania sp.), and Mu-Xiang (Radix aucklandiae) $[12,17]$.

The primary outcomes of this study included death attributable to all-cause or CKD-related diseases (defined by the aforementioned ICD-9-CM codes of CKD). To allow for at least one year of follow-up, all diagnoses of CKD had to be made before December 31, 2007. Subjects were followed from the date of first CKD diagnosis until death, loss of follow-up, or December 31, 2008, whichever came first.

2.3. Assessment of Exposure to Potentially Renoprotective Chinese Herbal Medicines ( $R P C H M s$ ). In Taiwan, all CHMs prescribed by licensed Chinese medicine physicians are eligible for reimbursement by the NHI Bureau. To assess the potential effects of RPCHMs, we identified eight CHMs reimbursed by the NHI Bureau that have been shown to have beneficial effects on renal function in clinical and animal studies, namely Cordyceps sinensis, da huang (Rheum palmatum L.), Astragalus membranaceus, dan shen (Salvia miltiorrhiza Bge.), Angelica sinensis, Saireito (Chai-Ling-Tang, a mixture herbal formulation) $[18,19]$, Yishen capsule (an encapsulated herbal formulation) [20], and WenPi Tang (a mixture herbal formulation) [21]. Because these CHMs might be prescribed as single constituents or compound CHM formulations, it was extremely difficult to calculate the exact dose of each CHM. Therefore, we categorized the treatment duration into nonuse and use of RPCHM. All RPCHMs use was further treated as a time-dependent variable to avoid immortal time bias [22]. 
2.4. Assessment of Potential Confounders. We collected the following variables to control the effects of potential confounders, namely, age; gender; comorbidities prior to $\mathrm{CKD}$ diagnoses, including hypertension, diabetes mellitus (DM), and cardiovascular disease (CVD); prior hospitalization (for any reason); use of nonsteroidal anti-inflammatory drugs (NSAIDs) and analgesics and the use of erythropoietin therapy. We classified age into four groups [3]: less than 40, $40-55,56-70$, and greater than 70 years.

The diagnosis of comorbidities was determined by searching for three consecutive outpatient visits or any hospitalization for the ICD-9-CM codes within three years before the CKD was diagnosed. Charlson's Comorbidity Index (CCI) was also employed to evaluate the overall disease burden of various comorbidities on each study subject [23]. The definitions of comorbidities other than hypertension, DM, and CVD were similar to those of the aforementioned diseases. The records of any hospitalization within three years prior to the diagnosis of CKD were treated as a potential confounder in data analysis as well.

Habitual smoking and drinking are potential confounders, but no data is available in LHID2000. To control for their confounding effects, we employed the diagnosis of chronic obstructive pulmonary disease (COPD) and alcoholic liver diseases as surrogates for habitual smoking and drinking, respectively. Moreover, we evaluated the study subjects' socioeconomic status based on their monthly wage. According to the quartile distribution of subjects' monthly wages, study subjects were classified into three groups: monthly wage lower than 15,840 New Taiwan dollars (NTD, equivalent to approximately 500 United States dollars [USD], the minimum monthly wage of full-time employees in Taiwan during the study period), between NTD 15,841 and 19,200 (approximately USD 600), and more than NTD 19,200.

We also assessed the dose of NSAIDs, analgesics, erythropoietin, and vitamin D supplements because these drugs are likely to confound the relationship between the use of RPCHMs and the risk of mortality in patients with CKD. The cumulative dose of each drug given to the subjects was then calculated by the number of prescribed pills. For NSAIDs and analgesics, we divided subjects by the annual total number of pills received within the previous year into $\leqq 104$ and >104 pills [24].

Erythropoietin was used as a surrogate marker of the severity of CKD in Taiwan because its use is strictly limited by the NHI Bureau to ESRD patients who have a hematocrit (Hct) value lower than $28 \%$ or CKD patients with serum creatinine over $6 \mathrm{mg} / \mathrm{dL}$ and Hct value less than 28\%. The use of erythropoietin and vitamin D supplements was classified as use or nonuse in this study.

2.5. Statistical Analysis. Log-rank test and Holm-Sidak multiple comparisons were used to examine the survival rates between different RPCHM treatment groups. Furthermore, a Cox proportional hazards regression model was used to evaluate the association between potential risk factors and CKD subjects' survival rate. All variables with a $p$ value less than 0.05 in univariate analyses were entered into the multivariate analysis, and a stepwise procedure was used to eliminate those variables that became insignificant. In addition, to determine whether the association between RPCHM use and survival status varied across different subgroups, we conducted subgroup analyses by stratifying subjects by age, gender, DM, hypertension, use of NSAIDs and analgesics, use of erythropoietin therapy, and prior hospitalization. All statistical analyses were performed with the SAS statistical package version 9.1.3 (SAS Institute, Inc., Cary, NC, USA). A $p$ value less than 0.05 was considered statistically significant. The study protocol of this retrospective cohort study was approved by the Institutional Review Board (IRB) of Landseed Hospital (Landseed IRB-12-23).

\section{Results}

Within the database, 39,003 patients were found to have incident CKD after January 1, 2000. Among them, 25,780 patients had ever received AA-containing CHMs and were thus excluded. Forty-nine patients were of a foreign nationality, and 1,498 patients had also been diagnosed with cancer or HIV. Moreover, 51 patients died within 1 month of the CKD diagnosis. After excluding these patients, a total of 11,625 CKD patients were eligible for further analysis (Figure 1).

The mean age of the study subjects was $55.1 \pm 20.9$ years. A total of 848 patients died during the follow-up period, including 279 (33\%) CKD-related deaths. Older age was associated with an increased risk of mortality (Table 1).

Deaths occurred more frequently in patients with various comorbidities, including hypertension, DM, CVD, higher CCI score, alcoholic liver disease, and COPD. The use of more than 104 pills of NSAIDs or analgesics per year after the diagnosis of CKD was also associated with all-cause mortality, but not with CKD-related mortality. Receipt of erythropoietin therapy was correlated with an increased risk of both all-cause and CKD-related mortality (Table 1).

In the analysis of RPCHMs, we initially sought to analyze the association between the use of each RPCHM and the risk of mortality after the diagnosis of CKD. However, only 1,919 subjects had RPCHMs prescribed after being diagnosed with CKD, and most of them $(n=1,381 ; 72 \%)$ received RPCHMs that contain Angelica sinensis. Therefore, we combined those patients who did not receive Angelica sinensis after the diagnosis of CKD into a group to allow more meaningful statistical analyses. The use of RPCHMs after the diagnosis of CKD was eventually classified into nonuse of RPCHMs, use of RPCHMs containing Angelica sinensis, and use of other RPCHMs. Use of RPCHMs before the diagnosis of CKD was associated with a low risk of all-cause mortality in bivariate analysis, whereas RPCHM use after the diagnosis of CKD was inversely correlated with both all-cause and CKD-related mortality (Table 1). Further analysis of the survival curve revealed that the use of RPCHMs (no matter containing Angelica sinensis or not) was generally associated with a lower risk of all-cause mortality compared to nonusers (Figure 2).

In contrast, only subjects who had received Angelica sinensis exhibited a better survival in CKD-related mortality than nonusers (Figure 3). 


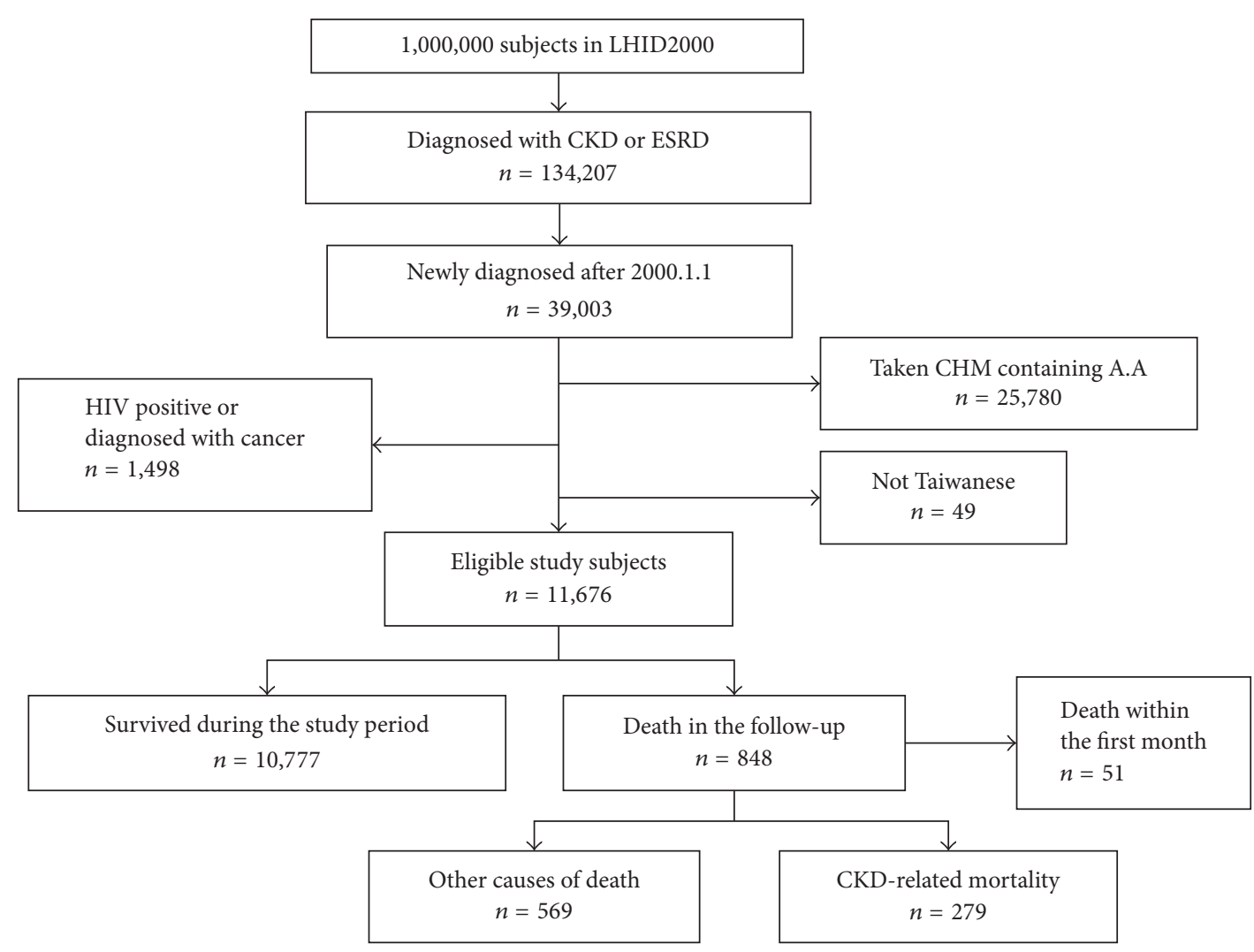

FIGURE 1: Patients' selections flow chart. AA: aristolochic acid; CHM: Chinese herbal medicines; CKD: chronic kidney disease; ESRD: endstage renal disease; HIV: human immunodeficiency virus.

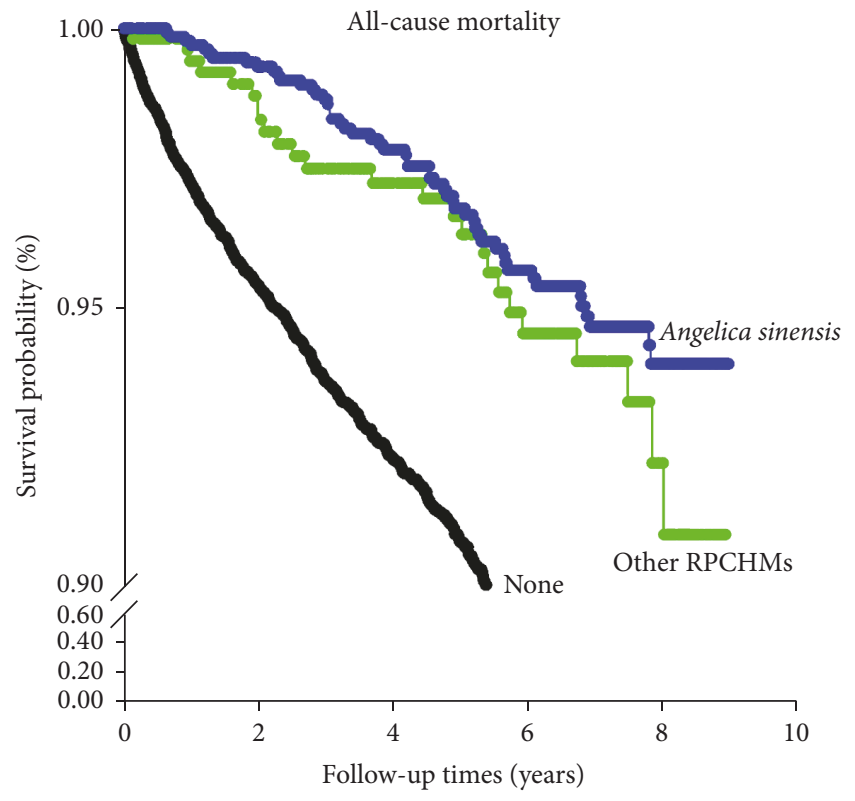

FIGURE 2: Survival curve for all-cause mortality among 11,625 patients with chronic kidney disease. RPCHM: renoprotective Chinese herbal medicines. Log-rank test (Holm-Sidak method): Angelica sinensis versus none, $p<0.001$; Angelica sinensis versus other RPCHMs, $p=0.263$; other RPCHMs versus none, $p<0.001$.
After controlling for potential confounding variables, the adjusted hazard ratio (aHR) for the use of other RPCHMs was 0.6 (95\% confidence interval [CI] 0.4-0.9, $p=0.013$ ), whereas the aHR for the use of Angelica sinensis was 0.6 (95\% CI 0.4-0.8, $p<0.001$ ) (Table 2). The relation between the use of Angelica sinensis and a lower risk of CKD-related mortality in bivariate analysis did not change after controlling for confounding variables with the aHR being 0.6 (95\% CI 0.4-0.9, $p=0.025$ ) (Table 2).

We also conducted subgroup analyses to see whether the findings varied across subgroups. Although the HR estimates were not available for certain subgroups because of limited sample size, the inverse association between the use of RPCHMs containing Angelica sinensis and the risk of mortality among CKD patients persisted across various subgroups. Nevertheless, the use of Angelica sinensis was not associated with reduced mortality in patients required erythropoietin therapy and use of NSAID or analgesics over 104 pills per year (Table 3 ).

\section{Discussion}

CKD is a worldwide public health problem and is recognized as a risk factor for cardiovascular events, hospitalization, and all-cause mortality $[5,25,26]$. The therapeutic strategies 
TABLE 1: Distribution of baseline characteristics between 10,777 alive subjects and 848 deceased subjects who had incident chronic kidney disease.

\begin{tabular}{|c|c|c|c|c|c|}
\hline \multirow[b]{2}{*}{ Variables } & \multirow{2}{*}{$\begin{array}{l}\text { Survival }(n, \%) \\
\quad(n=10777)\end{array}$} & \multicolumn{4}{|c|}{ Death $(n, \%)$} \\
\hline & & $\begin{array}{l}\text { All-cause } \\
(n=848)\end{array}$ & $p$ value & $\begin{array}{c}\text { CKD related } \\
(n=279)\end{array}$ & $p$ value \\
\hline \multicolumn{6}{|l|}{ Gender } \\
\hline Female & $4062(37.7)$ & $295(34.8)$ & \multirow[t]{2}{*}{0.092} & $116(41.6)$ & \multirow[t]{2}{*}{0.186} \\
\hline Male & $6715(62.3)$ & $553(65.2)$ & & $163(58.4)$ & \\
\hline Age (year, mean $\pm S D$ ) & $53.8 \pm 20.8$ & $71.8 \pm 14.1$ & $<0.001$ & $69.9 \pm 14.2$ & $<0.001$ \\
\hline$<40$ & $2718(25.2)$ & $23(2.7)$ & \multirow[t]{4}{*}{$<0.001$} & $9(3.2)$ & \multirow[t]{4}{*}{$<0.001$} \\
\hline $40-55$ & $2603(24.2$ & $89(10.5)$ & & $39(14.0)$ & \\
\hline $56-70$ & $2663(24.7)$ & $188(22.2)$ & & $65(22.3)$ & \\
\hline$>70$ & $2793(25.9)$ & $548(64.6)$ & & $166(59.5)$ & \\
\hline \multicolumn{6}{|l|}{ Monthly wage (NTD) } \\
\hline$\leq 15840$ & $4774(44.3)$ & $584(68.9)$ & \multirow[t]{3}{*}{$<0.001$} & $186(66.7)$ & \multirow[t]{3}{*}{$<0.001$} \\
\hline $15841-19200$ & $3764(34.9)$ & $220(25.9)$ & & $79(28.3)$ & \\
\hline$>19200$ & $2239(20.8)$ & $44(5.2)$ & & $14(5.0)$ & \\
\hline Hypertension & $5212(48.4)$ & $656(77.4)$ & $<0.001$ & $220(78.9)$ & $<0.001$ \\
\hline Diabetes mellitus & $3257(30.2)$ & $407(48.0)$ & $<0.001$ & $148(53.1)$ & $<0.001$ \\
\hline Cardiovascular disease ${ }^{*}$ & $2466(22.9)$ & $329(38.8)$ & $<0.001$ & $102(36.6)$ & $<0.001$ \\
\hline \multicolumn{6}{|l|}{ CCI } \\
\hline 0 & $2633(24.4)$ & $94(11.1)$ & \multirow[t]{3}{*}{$<0.001$} & $39(14.0)$ & \multirow[t]{3}{*}{$<0.001$} \\
\hline 1 & $2299(21.3)$ & $131(15.5)$ & & $37(13.2)$ & \\
\hline$\geqq 2$ & $5845(54.2)$ & $623(73.4)$ & & $203(72.8)$ & \\
\hline Ever being hospitalized before CKD & $1009(9.4)$ & $216(25.5)$ & $<0.001$ & $109(39.1)$ & $<0.001$ \\
\hline COPD & $1540(14.3)$ & $278(32.8)$ & $<0.001$ & $76(27.2)$ & $<0.001$ \\
\hline Alcoholic liver disease & $1409(13.1)$ & $132(15.6)$ & 0.039 & $47(16.9)$ & 0.066 \\
\hline Use of analgesics or NSAIDs > 104 pills/year & $373(3.5)$ & $53(6.3)$ & $<0.001$ & $15(5.4)$ & 0.086 \\
\hline Receipt of erythropoietin & $539(5.0)$ & $97(11.4)$ & $<0.001$ & $52(18.6)$ & $<0.001$ \\
\hline Receipt of vitamin D & $109(1.0)$ & $12(1.4)$ & 0.264 & $6(2.1)$ & 0.071 \\
\hline \multicolumn{6}{|l|}{ Current RPCHM use } \\
\hline None & $8936(82.9)$ & $770(90.8)$ & \multirow[t]{3}{*}{$<0.001$} & $253(90.7)$ & \multirow[t]{3}{*}{0.004} \\
\hline Other RPCHMs & $513(4.8)$ & $25(3.0)$ & & $9(3.2)$ & \\
\hline Angelica sinensis & $1328(12.3)$ & $53(6.2)$ & & $17(6.1)$ & \\
\hline Ever use of RPCHMs & $2046(19.0)$ & $127(15.0)$ & 0.004 & $41(14.7)$ & 0.070 \\
\hline
\end{tabular}

CCI: Charlson’s Comorbidity Index; CKD: chronic kidney disease; COPD: chronic obstructive pulmonary disease; NSAIDs: nonsteroidal anti-inflammatory drugs; NTD: New Taiwan dollars; RPCHMs: renoprotective Chinese herbal medicines. ${ }^{*}$ Including congenital heart disease.

for treating CKD are generally aimed at ameliorating the progression of CKD and preventing complications. Previous studies found that the use of AA-containing or nonprescribed CHMs might lead to CKD or ESRD [10, 12]. Therefore, although animal and clinical studies have demonstrated potential renoprotective effects of certain CHMs [18], most physicians tend to persuade patients with CKD to stop taking CHMs to prevent further deterioration of their renal function. A recent study however showed that patients with CKD who used prescribed CHMs exhibited a reduced risk of ESRD [14]. In a pilot study, we also found that patients with CKD in Taiwan who started to receive non-AA prescribed CHMs after the diagnosis of incident CKD had a lower risk of mortality compared with nonusers of non-AA prescribed
CHMs (aHR 0.6; 95\% CI 0.4 to 0.7) [11]. Moreover, patients with CKD who had received non-AA prescribed CHMs both prior to and after the diagnosis of CKD also had a lower risk of mortality than nonusers (aHR 0.6; 95\% CI 0.5 to 0.8 ) after the onset of CKD. In another study, the use of CHMs was independently associated with a decreased risk of renal failure among type 2 diabetic patients $(\mathrm{aOR}=0.7,95 \%$ CI $0.6-0.8)$ $(\mathrm{aOR}=0.7,95 \%$ CI 0.6-0.8) [11].

In this population-based follow-up study, we found that most prescribed RPCHMs were inversely associated with the risk of all-cause mortality among patients with CKD. Moreover, the use of RPCHMs containing Angelica sinensis after the diagnosis of CKD was associated with a lower risk of CKD-related mortality. The possible renoprotective effects 
TABLE 2: Multivariate Cox proportional hazards regression analysis of all-cause and CKD-related mortality of 11,625 CKD patients.

\begin{tabular}{|c|c|c|c|c|c|c|}
\hline \multirow{2}{*}{ Variables } & \multicolumn{3}{|c|}{ All-cause mortality } & \multicolumn{3}{|c|}{ CKD related mortality } \\
\hline & $\mathrm{HR}$ & $95 \% \mathrm{CI}$ & $p$ value & $\mathrm{HR}$ & $95 \% \mathrm{CI}$ & $p$ value \\
\hline Gender (male) & 1.2 & $1.1-1.4$ & 0.007 & 1.0 & $0.8-1.3$ & 0.937 \\
\hline \multicolumn{7}{|l|}{ Age (year) } \\
\hline$<40$ & Reference & - & - & Reference & - & - \\
\hline $40-55$ & 4.3 & $2.7-6.9$ & $<0.001$ & 4.2 & $2.0-8.9$ & $<0.001$ \\
\hline $56-70$ & 6.4 & $4.1-10.0$ & $<0.001$ & 4.6 & $2.2-9.6$ & $<0.001$ \\
\hline$>70$ & 14.7 & $9.4-23.0$ & $<0.001$ & 10.1 & $4.9-20.8$ & $<0.001$ \\
\hline \multicolumn{7}{|l|}{ Monthly wage (NTD) } \\
\hline$\leq 15840$ & Reference & - & - & Reference & - & - \\
\hline $15841-19200$ & 0.6 & $0.5-0.7$ & $<0.001$ & 0.6 & $0.5-0.8$ & $<0.001$ \\
\hline$>19200$ & 0.4 & $0.3-0.5$ & $<0.001$ & 0.3 & $0.2-0.6$ & $<0.001$ \\
\hline Hypertension & 1.4 & $1.2-1.7$ & $<0.001$ & 1.6 & $1.2-2.2$ & 0.004 \\
\hline Diabetes mellitus & 1.2 & $1.1-1.4$ & 0.005 & 1.3 & $1.0-1.7$ & 0.027 \\
\hline Ever being hospitalized before CKD & 2.5 & $2.1-2.9$ & $<0.001$ & 4.5 & $3.5-5.8$ & $<0.001$ \\
\hline COPD & 1.2 & $1.0-1.4$ & 0.014 & - & - & - \\
\hline Use of NSAIDs or analgesics $>104$ pills/year & 1.8 & $1.4-2.4$ & $<0.001$ & 1.7 & $1.0-2.9$ & 0.043 \\
\hline Receipt of erythropoietin & 1.4 & $1.1-1.7$ & 0.007 & 1.8 & $1.3-2.5$ & $<0.001$ \\
\hline \multicolumn{7}{|l|}{ Current RPCHM use } \\
\hline None & Reference & - & - & Reference & - & - \\
\hline Other RPCHMs & 0.6 & $0.4-0.9$ & 0.013 & 0.7 & $0.4-1.4$ & 0.346 \\
\hline Angelicasinensis & 0.6 & $0.4-0.8$ & $<0.001$ & 0.6 & $0.4-0.9$ & 0.025 \\
\hline Ever use of PRCHMs & 1.0 & $0.8-1.2$ & 0.790 & 0.9 & $0.7-1.3$ & 0.722 \\
\hline
\end{tabular}

CCI: Charlson's Comorbidity Index; CI: confidence interval; CKD: chronic kidney disease; COPD: chronic obstructive pulmonary disease; HR: hazard ratio; NTD: New Taiwan dollars; RPCHMs: renoprotective Chinese herbal medicines.

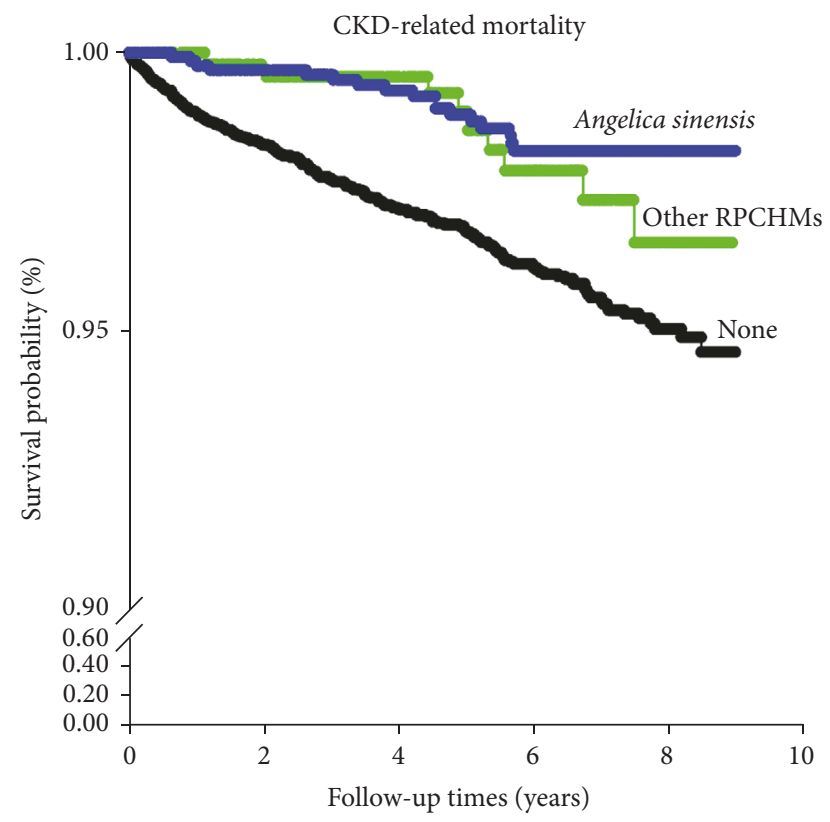

FIGURE 3: Survival curve for chronic kidney disease related mortality among 11,625 patients with chronic kidney disease. RPCHM: renoprotective Chinese herbal medicines. Log-rank test (Holm-Sidak method): Angelica sinensis versus none, $p<0.001$; Angelica sinensis versus other RPCHMs, $p=0.064$; other RPCHMs versus none, $p=0.351$. of RPCHMs largely persisted in subgroup analyses, but the seemingly beneficial effect disappeared among patients who used NSAIDs or analgesics more than 104 pills per year or who already had severe CKD, as indicated by the receipt of erythropoietin therapy.

Angelica sinensis is commonly used in CHM to enrich blood and promote blood circulation [27]. It has been found to have anti-inflammatory effects and can improve immune function by increasing the expression of cytokines in splenocytes and the production of interleukin- 6 and interferon- $\gamma$ of activated macrophages, helper T cells, and natural killer cells [28]. A previous study reported that the combined use of Angelica sinensis and other CHMs such as Astragalus membranaceus and Astragali radix exerted antifibrosis effects and were associated with improvements in ischemic microvasculature and renal interstitial fibrosis [29].

The major ingredients of Angelica sinensis include ligustilide, n-butylidene phthalide, ferulic acid, nicotinic acid, and sesquiterpenoid compounds [30]. These ingredients have been found to possess analgesic, anti-inflammatory, immunomodulatory, and even anticancer effects [31, 32]. Among them, ligustilide is the major active component of Angelica sinensis and has many pharmaceutical effects such as vasodilatation [33] and inhibition of the proliferation of vascular smooth muscle cells to retard vascular sclerosis [34]. Therefore, Angelica sinensis may have beneficial effects on the 


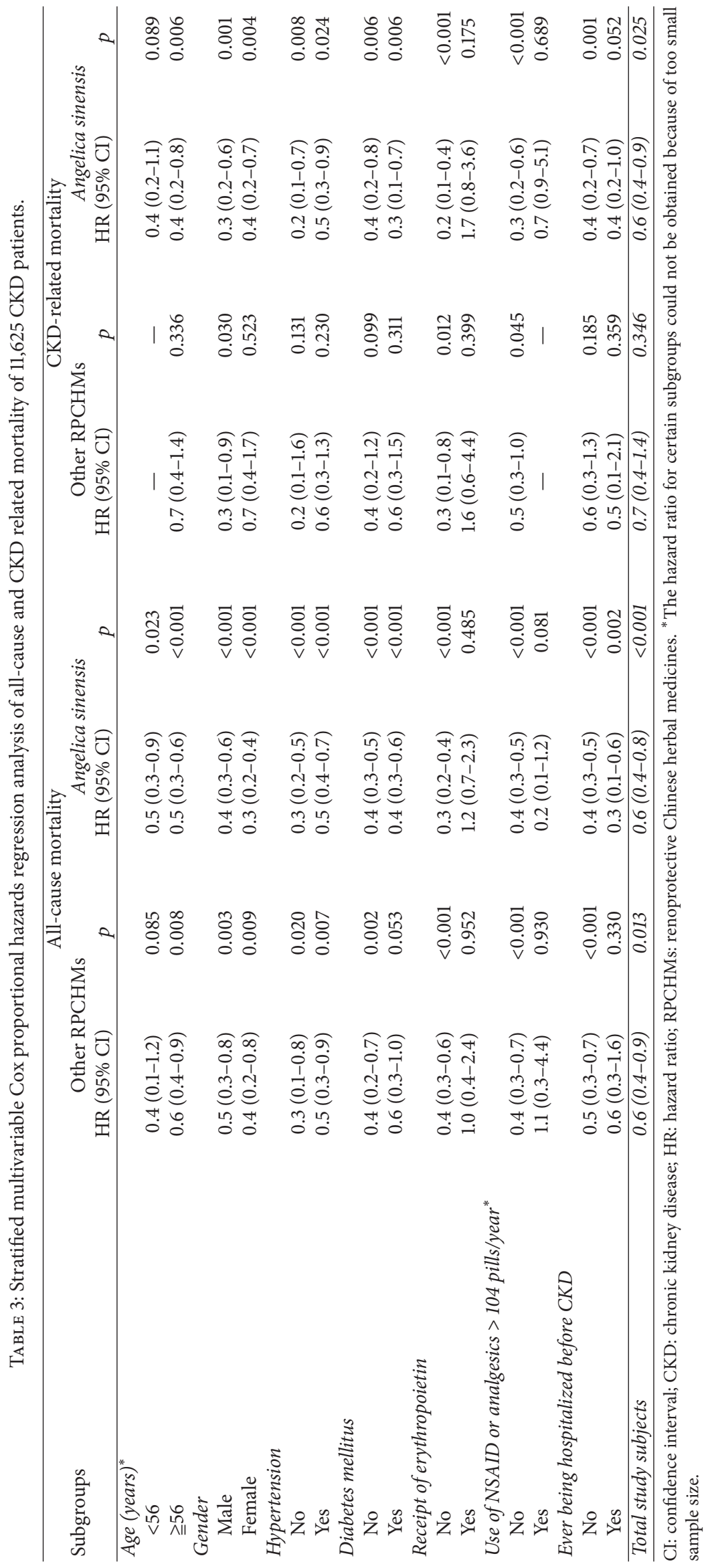


progression of CKD and subsequently reduce the mortality of CKD patients.

In addition to Angelica sinensis, the use of other RPCHMs was associated with a lower risk of all-cause mortality. Among the other RPCHMs evaluated in this study, Astragalus membranaceus is also commonly used in CHM. It is usually prescribed in conjunction with Angelica sinensis, a combination therapy that has been found to inhibit renal tubulointerstitial fibrosis in rats with obstructive nephropathy $[19,35]$.

Similarly, Shahed et al. found that Cordyceps sinensis exhibited potent antioxidant activity and protected the kidneys by inhibiting mesangial cell proliferation [36]. In a clinical trial, long-term use of Cordyceps sinensis for up to 3 years led to reduced levels of creatinine and blood urea nitrogen (BUN) in patients with lupus nephritis [37]. In another trial, Cordyceps sinensis displayed a protective role in aminoglycoside induced nephrotoxicity in the elderly [38].

The use of 6 to 9 grams of Rheum palmatum per day in patients with CKD was also found to effectively delay the progression of CKD to ESRD [39]. Salvia miltiorrhiza contains at least seven phenolic compounds with strong antioxidant activities that were effective in reducing BUN and eventual renoprotection in rats with impaired renal function [40]. Salvia miltiorrhiza was also shown to improve platelet function and regulation of coagulation in dialysis patients [41].

Yishen capsule was effective in reducing the production of proteinuria in patients with chronic glomerulonephritis [42]; improving serum creatinine, BUN and cholesterol; and consequently ameliorating the progression to ESRD [20]. In addition, many Japanese studies have indicated that Saireito and Wen Pi Tang could lower the levels of serum creatinine, urine protein, and systolic blood pressure, thus making them effective in delaying the progression of CKD to ESRD [21, 43, 44].

We did observe an inverse association between the use of RPCHMs other than Angelica sinensis and the risk of all-cause mortality in this study. However, we were unable to ascertain which of the other RPCHMs contributed to the beneficial effects on the mortality of patients with CKD because of the limited sample size of patients receiving individual RPCHMs. Further larger scale or prospective studies are warranted to delineate the renoprotective effects of various RPCHMs.

Similar to western medicine, the use of traditional herbal medicine including Angelica sinensis and other RPCHMs also may have undesirable effects if they are used without careful evaluation by the treating physicians [19]. For example, ferulic acid, an extract derived from Angelica sinensis, has adverse immunomodulatory effects in patients with certain hormone-sensitive conditions such as breast cancer or ovarian cancer [45]. Moreover, Astragalus membranaceus is well known to inhibit cytochrome P450 3A4 (CYP3A4) and can reduce cyclophosphamide-induced immunosuppression [46]. Long-term use of Rheum palmatum had been reported to cause electrolyte depletion and liver toxicity as well [47]. Therefore, although RPCHMs may be beneficial to patients with $\mathrm{CKD}$, initiation of treatment with potentially renoprotective CHMs should always be carefully evaluated by licensed Chinese medicine physicians.
Hypertension is a well-known risk factor for CKD and its progression. Poorly controlled blood pressure can lead to CKD deterioration and consequent CVD and mortality [48]. In the present study, we found that hypertension also played an important role in both all-cause mortality and CKD-related mortality. Apart from hypertension, DM is an important risk factor for CKD progression as well. According to the Diabetes Control and Complications Trial [49] and UK Prospective of Diabetes Study [50], patients with poorly controlled type $2 \mathrm{DM}$ had a higher chance of developing macroalbuminuria and progressively worsened nephropathy or even death. Our findings on DM are thus consistent with previous reports.

CVD is a major complication that can cause death in patients with CKD [25]. In this study, although CVD was a risk factor for mortality in the bivariate analysis (Table 1), it was not associated with the risk of mortality after controlling for other confounding variables. This is probably because of the presence of collinearity between $\mathrm{CKD}$ and other comorbidities, especially hypertension, DM [48, 51], and age. Similarly, CCI, an indicator used to assess the overall burden of disease in patients [23], also lost its statistical significance in multivariate Cox proportional hazards regression models.

Poverty affects the accessibility to medical treatment for patients with CKD and related mortality [52, 53]. Although the overwhelming majority of Taiwanese residents are included in the NHI program, patients must pay a nominal out-of-pocket copayment out of their own pockets for each clinic visit. Therefore, patients from poorer economic conditions might have less medical access and poor disease control, which could then lead to a higher risk of mortality.

Smoking was found to be an attributable risk in 31\% of CKD cases in a large cohort study [54]. Moreover, the risk of rapid progression to ESRD was 3.5 times higher in patients with CKD who smoked more than five packets of cigarettes per year [55]. As the information on smoking status was unavailable in the NHIRD, we employed COPD as a surrogate marker for smoking status and found a positive association between COPD and all-cause mortality. Although smoking accounts for 80 to $90 \%$ of the incidence of COPD, there are other etiologies of COPD such as air pollution, exposure to chemicals or irritating substances, dust, and allergens [56]. Therefore, the observed association between COPD and allcause mortality may or may not completely reflect the relation between smoking and mortality.

Some prior studies suggested that the use of NSAIDs and analgesics was associated with CKD progression, the development of ESRD, or death [24, 57]. However, not all studies supported the positive association between NSAID exposure and the risk of renal function loss [58, 59]. We found the use of $\geqq 104$ pills of NSAIDs or analgesics per year was associated with all-cause mortality and CKD-related mortality in patients with incident CKD, which supports the nephrotoxic potential of both NSAIDs and analgesic therapy.

Erythropoietin is a glycoprotein hormone with the function of regulating the formation of red blood cells in the human body and is commonly used to treat anemia following dialysis for patients with ESRD. In Taiwan, the use of erythropoietin is strictly limited to patients with ESRD who 
have a Hct value less than $28 \%$ or patients with CKD who have a serum creatinine greater than $6 \mathrm{mg} / \mathrm{dL}$ and a $\mathrm{Hct}$ value less than $28 \%$. Therefore, the use of erythropoietin was associated with the risk of all-cause mortality and CKDrelated mortality. Similarly, patients who were hospitalized prior to the diagnosis of CKD were also associated with the risk of mortality.

\section{Limitations}

We found that the use of prescribed RPCHMs, especially Angelica sinensis, was associated with a lower risk of mortality in patients with incident CKD. However, the study findings should be interpreted with caution, and a causal relation between the use of RPCHMs and the risk of mortality cannot be inferred based on such a retrospective follow-up study because of several methodological limitations. First, in this observational study, we are unable to completely exclude the possibility of unmeasured confounding effects. For example, several potential risk factors of CKD and related mortality are not available in LHID2000, such as smoking, alcohol drinking, family history of CKD, and exercise habits. These factors can thus confound the findings if they are also associated with the use of RPCHMs. Although we had used COPD and alcoholic liver disease as surrogate markers to control for the potential confounding effects of smoking and alcohol consumption, the lack of direct measurement on relevant variables might result in residual confounding.

The validity of LHID2000 may also be of concern because the database is a claims database that is not designed for research purposes. Nevertheless, the NHI program is the only nationwide insurance program in Taiwan and covers more than $96 \%$ of the Taiwanese residents. Furthermore, the accuracy of medical information recorded in LHID2000 has been validated by both the NHI Bureau and some researchers in Taiwan [60].

The information on serum creatinine or glomerular filtration rate is unavailable in LHID2000, so we were unable to stratify the data analyses by the stages of CKD. We therefore used erythropoietin as well as vitamin D therapy as indices of the severity of CKD. We found that the use of erythropoietin but not vitamin D therapy was significantly associated with the risk of both all-cause and CKD-related mortality. We further revealed that the inverse associations between the use of RPCHMs and mortality were not present among patients with CKD who already received erythropoietin.

We initially attempted to evaluate the beneficial effects of individual RPCHMs, but numerous RPCHM formulations are prescribed in Taiwan, and it is extremely difficult to calculate the cumulative dose of each one. Moreover, the number of patients receiving most RPCHMs was relatively limited. We therefore divided the use of RPCHMs into those containing Angelica sinensis and those that do not. This led to a difficulty in disentangling the potential renoprotective effect across various RPCHMs. We were also unable to compare the difference in renoprotective effect between the use of Angelica sinensis alone and the consumption of Angelica sinensis with other RPCHMs because Angelica sinensis was almost always prescribed concomitantly with other RPCHMs in this study.
Further larger scale and prospective studies are warranted to better delineate the renoprotective effects of individual RPCHM and the potential interactions between different RPCHMs.

Selection bias is still possible in this retrospective population-based follow-up study. To minimize such bias, we excluded patients who had died within the first month after the diagnosis of incident CKD because they were less likely to receive RPCHM treatment for their illness. However, unforeseen differences in the baseline characteristics between patients who received RPCHMs after CKD diagnosis and those who did not could still be present and account for the findings of this study.

Finally, information related to the use of nonprescribed CHMs is not included in LHID2000. In a previous casecontrol study, we found that $6.6 \%$ of the controls had received nonprescribed CHMs, and the use of nonprescribed CHMs was positively associated with the risk of CKD [10]. We speculated that such an association was probably attributable to the adulteration of nonprescribed CHMs by pharmaceuticals. In Taiwan, the NHI Bureau mandates that all reimbursed CHMs be manufactured following "Good Manufacturing Practice (GMP)." There was also no significant relationship between the use of nonprescribed and prescribed CHMs among patients with CKD in a previous study [10]. Therefore, the use of nonprescribed CHMs was less likely to contribute to the observed inverse association between RPCHMs and the risk of mortality.

\section{Conclusions}

We found that the use of certain RPCHMs after the diagnosis of CKD, especially Angelica sinensis, was associated with a lower risk of all-cause and CKD-related mortality among patients with incident CKD. However, such an association was not observed among patients with severe CKD who required erythropoietin therapy.

\section{Conflicts of Interest}

The authors declare that they have no conflicts of interest.

\section{Authors' Contributions}

Chuan-Fa Hsieh and Huan-Cheng Chang contributed equally to this work.

\section{References}

[1] P. M. Barnes, E. Powell-Griner, K. McFann, and R. L. Nahin, "Complementary and alternative medicine use among adults: United States, 2002," Advance Data, no. 343, pp. 1-19, 2004.

[2] WHO Regional Office for the Western Pacific, The Regional Strategy for Traditional Medicine in The Western Pacific (2011-2020), http://www.wpro.who.int/publications/PUB_ 9789290615590/en/.

[3] M. N. Lai, J. N. Lai, and P. C. Chen, "Increased risks of chronic kidney disease associated with prescribed Chinese herbal products suspected to contain aristolochic acid," Nephrology, vol. 14, no. 2, pp. 227-234, 2009. 
[4] J. L. Vanherweghem, M. Depierreux, C. Tielemans et al., "Rapidly progressive interstitial renal fibrosis in young women: association with slimming regimen including Chinese herbs," The Lancet, vol. 341, no. 8842, pp. 387-391, 1993.

[5] C. P. Wen, T. Y. D. Cheng, M. K. Tsai et al., "All-cause mortality attributable to chronic kidney disease: a prospective cohort study based on 462293 adults in Taiwan," The Lancet, vol. 371, no. 9631, pp. 2173-2182, 2008.

[6] H. Y. Chen, B.-Y. Ma, A. Grant, and N. Lampert, "Time to abandon the term "Chinese herbs nephropathy"," Kidney International, vol. 60, no. 5, pp. 2039-2040, 2001.

[7] X. M. Li and H. Y. Wang, "Chinese herbal medicine in the treatment of chronic kidney disease," Advances in Chronic Kidney Disease, vol. 12, no. 3, pp. 276-281, 2005.

[8] A. Peng, Y. Gu, and S. Y. Lin, "Herbal treatment for renal diseases," ANNALS Academy of Medicine Singapore, vol. 34, pp. $44-51$.

[9] N. R. Burrows, Y. Li, and D. E. Williams, "Racial and ethnic differences in trends of end-stage renal disease: United States, 1995 to 2005," Advances in Chronic Kidney Disease, vol. 15, no. 2 , pp. 147-152, 2008.

[10] C.-F. Hsieh, S.-L. Huang, C.-L. Chen et al., "Increased risk of chronic kidney disease among users of non-prescribed Chinese herbal medicine in Taiwan," Preventive Medicine, vol. 55, no. 2, pp. 155-159, 2012.

[11] C. F. Hsieh, S. L. Huang, C. L. Chen, W. T. Chen, H. C. Chang, and C. C. Yang, "Non-aristolochic acid prescribed Chinese herbal medicines and the risk of mortality in patients with chronic kidney disease: Results from a population-based follow-up study," BMJ Open, vol. 4, no. 2, Article ID e004033, 2014.

[12] M. N. Lai, J. N. Lai, P. C. Chen, S. H. Hsieh, F. C. Hu, and J. D. Wang, "Risks of kidney failure associated with consumption of herbal products containing Mu Tong or Fangchi: a populationbased case-control study," American Journal of Kidney Diseases, vol. 55, no. 3, pp. 507-518, 2010.

[13] P.-C. Hsu, Y. Tsai, J. Lai, C. Wu, S. Lin, and C. Huang, "Integrating traditional Chinese medicine healthcare into diabetes care by reducing the risk of developing kidney failure among type 2 diabetic patients: a population-based case control study," Journal of Ethnopharmacology, vol. 156, pp. 358-364, 2014.

[14] M.-Y. Lin, Y.-W. Chiu, J.-S. Chang et al., "Association of prescribed Chinese herbal medicine use with risk of end-stage renal diseaseh chronic kidney disease," Kidney International, vol. 88, no. 6, pp. 1365-1373, 2015.

[15] E. K.-L. Lee, T.-M. Cham, and P.-L. Tseng, "Using the pharmacoepidemiology approach to evaluate the first-year posttransplantation ambulatory health care cost from the longitudinal health insurance database (2001 to 2006) in Taiwan," Transplantation Proceedings, vol. 42, no. 3, pp. 957-960, 2010.

[16] H.-W. Kuo, S.-S. Tsai, M.-M. Tiao, Y.-C. Liu, I.-M. Lee, and C.Y. Yang, "Analgesic use and the risk for progression of chronic kidney disease," Pharmacoepidemiology and Drug Safety, vol. 19, no. 7, pp. 745-751, 2010.

[17] M. N. Lai, S. M. Wang, P. C. Chen, Y. Chen, and J. Wang, "Population-based case-control study of chinese herbal products containing aristolochic acid and urinary tract cancer risk," Journal of the National Cancer Institute, vol. 102, no. 3, pp. 179$186,2010$.

[18] K. Wojcikowski, D. W. Johnson, and G. Gobe, "Herbs or natural substances as complementary therapies for chronic kidney disease: ideas for future studies," Journal of Laboratory and Clinical Medicine, vol. 147, no. 4, pp. 160-166, 2006.

[19] Y. Zhong, Y. Deng, Y. Chen, P. Y. Chuang, and J. Cijiang He, "Therapeutic use of traditional Chinese herbal medications for chronic kidney diseases," Kidney International, vol. 84, no. 6, pp. 1108-1118, 2013.

[20] G. Wang, C. Li, and L. Jin, "Experimental study on treatment of chronic renal failure with yishen qudu capsule," Zhongguo Zhong Xi Yi Jie He Za Zhi, vol. 20, no. 10, pp. 761-763.

[21] T. Mitsuma, T. Yokozawa, H. Oura, K. Terasawa, and M. Narita, "Clinical evaluation of kampo medication, mainly with wen-pitang, on the progression of chronic renal failure," Nihon Jinzo Gakkai Shi, vol. 41, no. 8, pp. 769-777.

[22] S. Z. Shariff, M. S. Cuerden, A. K. Jain, and A. X. Garg, "The secret of immortal time bias in epidemiologic studies," Journal of the American Society of Nephrology, vol. 19, no. 5, pp. 841-843, 2008.

[23] M. E. Charlson, P. Pompei, K. L. Ales, and C. R. MacKenzie, "A new method of classifying prognostic comorbidity in longitudinal studies: development and validation," Journal of Chronic Diseases, vol. 40, no. 5, pp. 373-383, 1987.

[24] T. V. Perneger, P. K. Whelton, and M. J. Klag, "Risk of kidney failure associated with the use of acetaminophen, aspirin, and nonsteroidal antiinflammatory drugs," New England Journal of Medicine, vol. 331, no. 25, pp. 1675-1679, 1994.

[25] A. S. Go, G. M. Chertow, D. Fan, C. E. McCulloch, and C. Hsu, "Chronic kidney disease and the risks of death, cardiovascular events, and hospitalization," The New England Journal of Medicine, vol. 351, no. 13, pp. 1296-1305, 2004.

[26] M. Tonelli, N. Wiebe, B. Culleton et al., "Chronic kidney disease and mortality risk: a systematic review," Journal of the American Society of Nephrology, vol. 17, no. 7, pp. 2034-2047, 2006.

[27] Q. Yang, S. M. Populo, J. Zhang, G. Yang, and H. Kodama, "Effect of Angelica sinensis on the proliferation of human bone cells," Clinica Chimica Acta, vol. 324, no. 1-2, pp. 89-97, 2002.

[28] S. B. Han, Y. H. Kim, C. W. Lee et al., "Characteristic immunostimulation by angelan isolated from Angelica gigas Nakai," Immunopharmacology, vol. 40, no. 1, pp. 39-48, 1998.

[29] L. Meng, L. Qu, J. Tang, S.-Q. Cai, H. Wang, and X. Li, "A combination of Chinese herbs, Astragalus membranaceus var. mongholicus and Angelica sinensis, enhanced nitric oxide production in obstructed rat kidney," Vascular Pharmacology, vol. 47, no. 2-3, pp. 174-183, 2007.

[30] L. Yi, Y. Liang, H. Wu, and D. Yuan, "The analysis of radix angelicae sinensis (Danggui)," Journal of Chromatography A, vol. 1216, no. 11, pp. 1991-2001, 2009.

[31] C. L. L. Saw, Q. Wu, Z.-Y. Su et al., "Effects of natural phytochemicals in Angelica sinensis (Danggui) on Nrf2-mediated gene expression of phase II drug metabolizing enzymes and anti-inflammation," Biopharmaceutics Drug Disposition, vol. 34, no. 6, pp. 303-311, 2013.

[32] A.-H. Zuo, L. Wang, and H.-B. Xiao, "Research progress studies on pharmacology and pharmacokinetics of ligustilide," Zhongguo Zhong Yao Za Zhi, vol. 37, no. 22, pp. 3350-3353, 2012.

[33] Y.-X. Cao, W. Zhang, J.-Y. He, L.-C. He, and C.-B. Xu, "Ligustilide induces vasodilatation via inhibiting voltage dependent calcium channel and receptor-mediated $\mathrm{Ca} 2+$ influx and release," Vascular Pharmacology, vol. 45, no. 3, pp. 171-176, 2006.

[34] Q. Lu, T.-Q. Qiu, and H. Yang, "Ligustilide inhibits vascular smooth muscle cells proliferation," European Journal of Pharmacology, vol. 542, no. 1-3, pp. 136-140, 2006. 
[35] L. Q. Meng, J. W. Tang, Y. Wang et al., "Astragaloside IV synergizes with ferulic acid to inhibit renal tubulointerstitial fibrosis in rats with obstructive nephropathy," British Journal of Pharmacology, vol. 162, no. 8, pp. 1805-1818, 2011.

[36] A. R. Shahed, S. I. Kim, and D. A. Shoskes, "Down-regulation of apoptotic and inflammatory genes by Cordyceps Sinensis extract in rat kidney following ischemia/reperfusion," Transplantation Proceedings, vol. 33, no. 6, pp. 2986-2987, 2001.

[37] L. Lu, "Study on effect of cordyceps sinensis and artemisinin in preventing recurrence of lupus nephritis," Zhongguo Zhong Xi Yi Jie He Za Zhi, vol. 22, no. 3, pp. 169-171.

[38] Z. D. Bao, Z. G. Wu, and F. Zheng, "Amelioration of aminoglycoside nephrotoxicity by Cordyceps sinensis in old patients," Zhongguo Zhong Xi Yi Jie He Za Zhi, vol. 14, no. 5, pp. 271-273.

[39] J. H. Zhang, L. S. Li, and M. Zhang, "Clinical effects of rheum and captopril on preventing progression of chronic renal failure," Chinese Medical Journal, vol. 103, no. 10, pp. 788-793.

[40] T. Yokozawa, J. J. Zhou, M. Hattori et al., "Effects of a Dan Shen component, magnesium lithospermate B, in nephrectomized rats," Nihon Jinzo Gakkai Shi, vol. 37, no. 2, pp. 105-111.

[41] Y. Li, L. Shen, R. Chen et al., "Effects of salivae miltiorrhizae liguspyragine hydrochloride and glucose injection the levels of main platelet thrombin receptors in chronic haemodialysis patients," Chinese Journal of Integrative Medicine, vol. 17, no. 8, pp. 625-630, 2011.

[42] X.-L. Wu, W.-S. Sun, W.-G. Zhang, C.-L. Qiao, Q.-Y. Ma, and X.-Q. Zhang, "Study on effect of Yishen capsule in preventing recurrence of chronic glomerulonephritis," Chinese Journal of Integrative Medicine, vol. 13, no. 3, pp. 215-218, 2007.

[43] L. He, P. Shen, Q. Fu et al., "Nephro-protective effect of kangqianling decoction on chronic renal failure rats," Journal of Ethnopharmacology, vol. 122, no. 2, pp. 367-373, 2009.

[44] J. Wang, Y. G. Wan, W. Sun, H. L. Zhang, P. Chen, and J. Yao, "Progress in Japanese herbal medicine in treatment of chronic kidney disease," Zhongguo Zhong Yao Za Zhi, vol. 33, no. 11, pp. 1348-1352.

[45] C. J. Chang, J. H. Chiu, L. M. Tseng et al., "Modulation of HER2 expression by ferulic acid on human breast cancer MCF7 cells," European Journal of Clinical Investigation, vol. 36, no. 8, pp. 588596, 2006.

[46] L. Guo, S.-P. Bai, L. Zhao, and X.-H. Wang, "Astragalus polysaccharide injection integrated with vinorelbine and cisplatin for patients with advanced non-small cell lung cancer: effects on quality of life and survival," Medical Oncology, vol. 29, no. 3, pp. 1656-1662, 2012.

[47] J. Yang, "Clinical use and side effects of Rheum palmatum L," China Practical Medicine, vol. 7, pp. 131-230.

[48] T. H. Jafar, P. C. Stark, C. H. Schmid et al., "Progression of chronic kidney disease: the role of blood pressure control, proteinuria, and angiotensin-converting enzyme inhibition: a patient-level meta-analysis," Annals of Internal Medicine, vol. 139, no. 4, pp. 244-252, 2003.

[49] T. D. C. a. C. D. R. Group, "Effect of intensive therapy on the development and progression of diabetic nephropathy in the diabetes control and complications trial: the diabetes control and complications (DCCT) research group," Kidney International, vol. 47, no. 6, pp. 1703-1720.

[50] A. L. Aldler, R. J. Steven, and S. E. Manley, "Development and progression of nephropathy in type 2 diabetes: the United Kingdom prospective diabetes study (UKPDS 64)," Kidney International, vol. 63, no. 1, pp. 225-232, 2003.
[51] M. R. Weir, "Recognizing the link between chronic kidney disease and cardiovascular disease," The American Journal of Managed Care, vol. 17, 15, pp. S396-S402, 2011.

[52] Y. N. Hall, A. I. Choi, G. M. Chertow, and A. B. Bindman, "Chronic kidney disease in the urban poor," Clinical Journal of the American Society of Nephrology, vol. 5, no. 5, pp. 828-835, 2010.

[53] R. Mehrotra and K. Norris, "Hypovitaminosis D, neighborhood poverty, and progression of chronic kidney disease in disadvantaged populations," Clinical Nephrology, vol. 74, 1, pp. S95-S98.

[54] M. K. Haroun, B. G. Jaar, S. C. Hoffman, G. W. Comstock, M. J. Klag, and J. Coresh, "Risk factors for chronic kidney disease: a prospective study of 23,534 men and women in Washington County, Maryland," Journal of the American Society of Nephrology, vol. 14, no. 11, pp. 2934-2941, 2003.

[55] S. R. Orth, A. Stockmann, C. Conradt et al., "Smoking as a risk factor for end-stage renal failure in men with primary renal disease," Kidney International, vol. 54, no. 3, pp. 926-931, 1998.

[56] R. A. Pauwels, A. S. Buist, P. M. A. Calverley, C. R. Jenkins, and S. S. Hurd, "Global strategy for the diagnosis, management, and prevention of chronic obstructive pulmonary disease: NHLBI/WHO global initiative for chronic obstructive lung disease (GOLD) workshop summary," American Journal of Respiratory and Critical Care Medicine, vol. 163, no. 5, pp. 12561276, 2001.

[57] K. Gooch, B. F. Culleton, B. J. Manns et al., "NSAID use and progression of chronic kidney disease," American Journal of Medicine, vol. 120, no. 3, pp. 280-e7, 2007.

[58] G. C. Curhan, E. L. Knight, B. Rosner, S. E. Hankinson, and M. J. Stampfer, "Lifetime nonnarcotic analgesic use and decline in renal function in women," Archives of Internal Medicine, vol. 164, no. 14, pp. 1519-1524, 2004.

[59] T. Kurth, R. J. Glynn, A. M. Walker et al., "Analgesic use and change in kidney function in apparently healthy men," American Journal of Kidney Diseases, vol. 42, no. 2, pp. 234-244, 2003.

[60] H.-C. Lin, Y.-J. Lin, T.-C. Liu, C.-S. Chen, and W.-T. Chiu, "Urbanization and stroke prevalence in Taiwan: analysis of a nationwide survey," Journal of Urban Health, vol. 84, no. 4, pp. 604-614, 2007. 


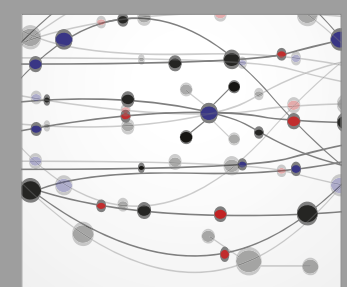

The Scientific World Journal
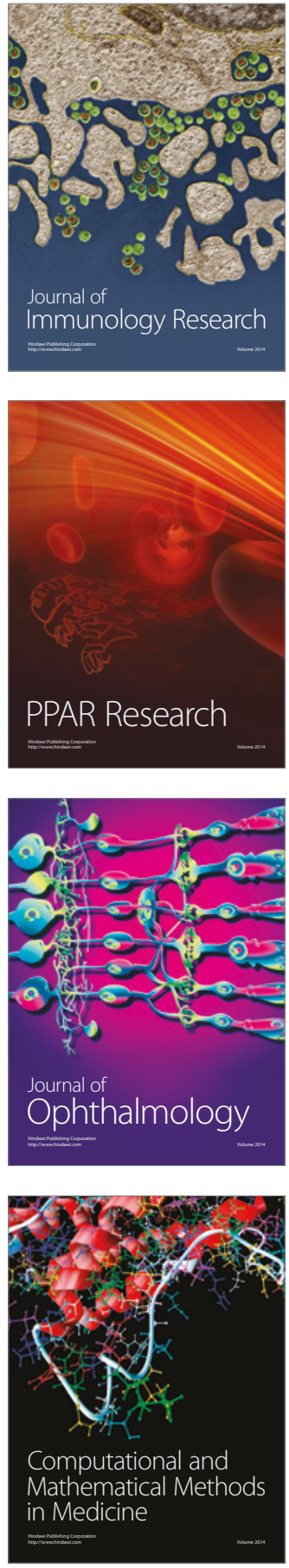

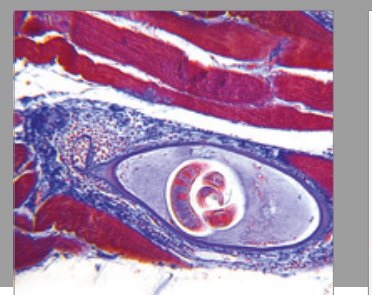

Gastroenterology Research and Practice
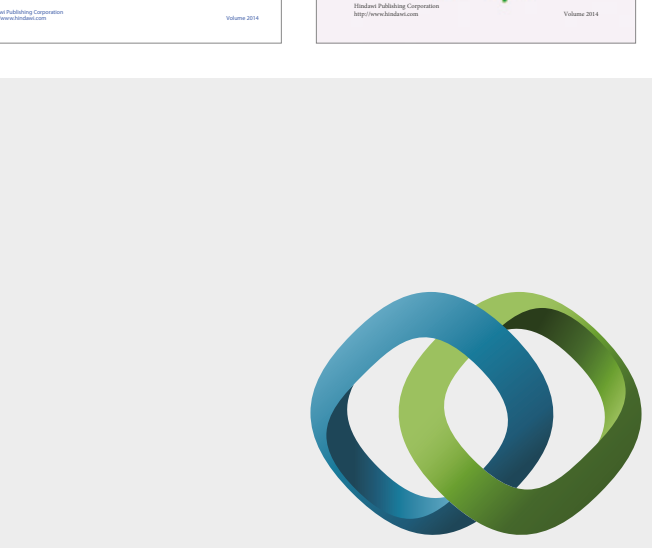

\section{Hindawi}

Submit your manuscripts at

https://www.hindawi.com
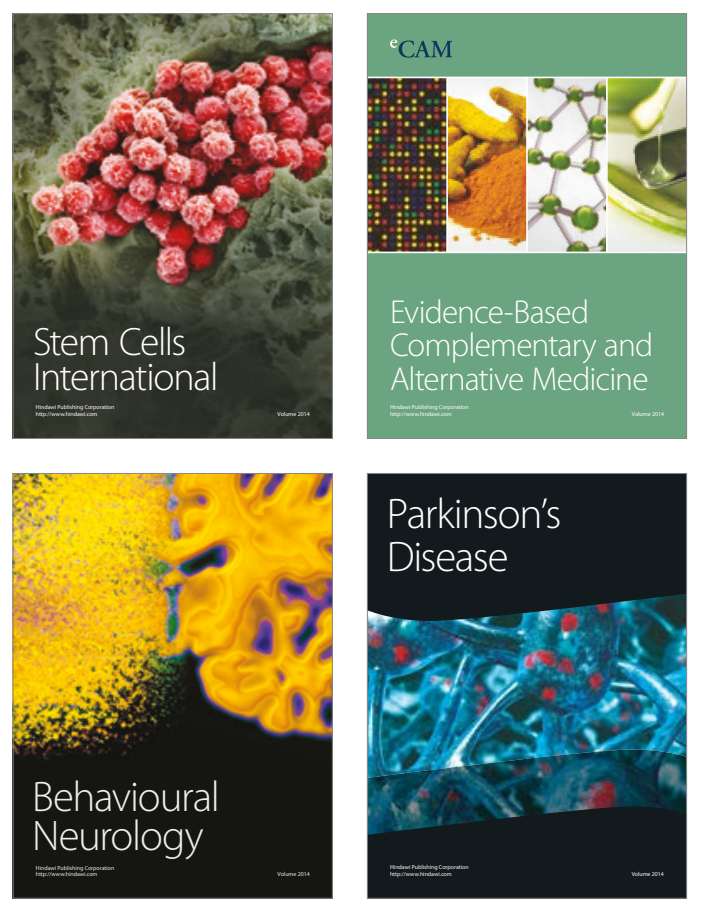
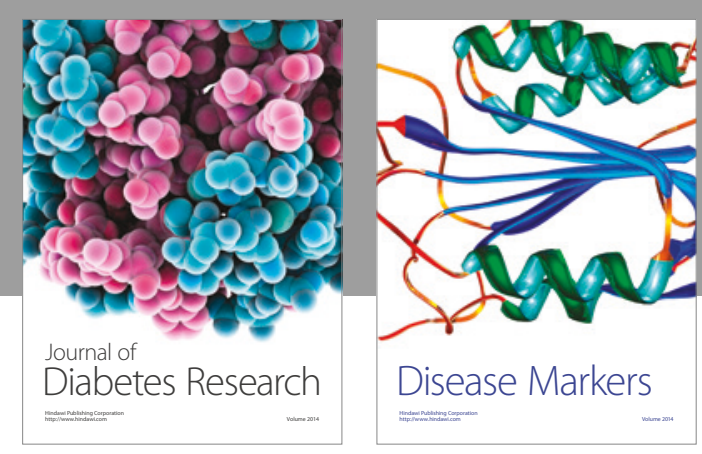

Disease Markers
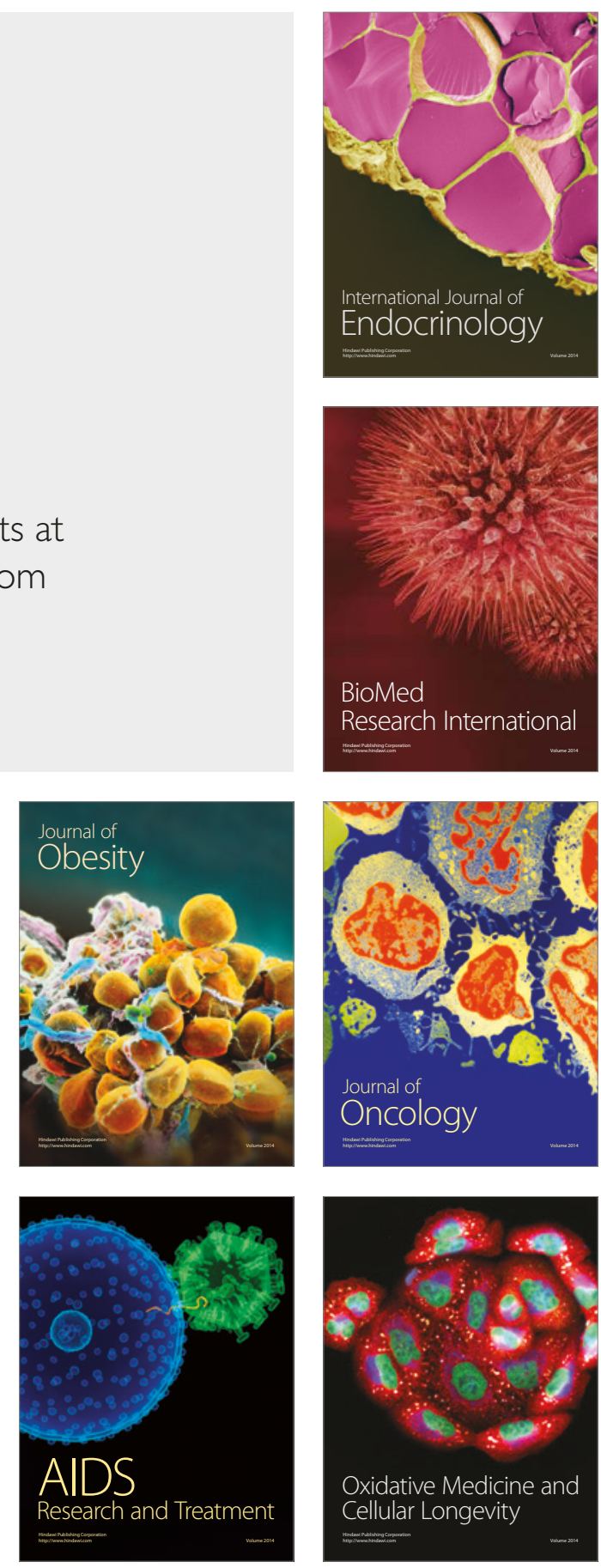